Acta Crystallographica Section F

Structural Biology

Communications

ISSN 2053-230X

\section{Shutao Xie}

MOE Key Laboratory of Protein Science, School of Life Sciences, Tsinghua University, Beijing 100084, People's Republic of China

Correspondence e-mail: xieshutao@sina.com

Received 13 May 2014 Accepted 19 June 2014

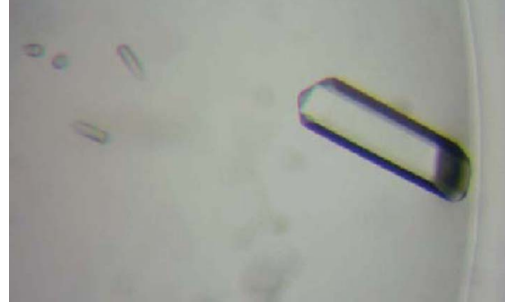

C 2014 International Union of Crystallography All rights reserved

\section{Characterization, crystallization and preliminary X-ray crystallographic analysis of the human Uba5 C-terminus-Ufc1 complex}

Human Uba5, which contains an adenylation domain and a C-terminal region, is the smallest ubiquitin-like molecule-activating enzyme. The mechanism through which the enzyme recognizes Ufc1 and catalyzes the formation of the Ufc1Ufm1 complex remains unknown. In this study, Uba5 residues 364-404 were demonstrated to be necessary for the transthiolation of Ufm1 to Ufc1, and Uba5 381-404 was identified to be the minimal region for Ufc1 recognition. The fusion protein between Uba5 381-404 and Ufc1 was cloned, expressed and purified, and exists as a homodimer in solution. Crystallization was performed at $293 \mathrm{~K}$ using PEG 4000 as precipitant; the optimized crystals diffracted to $3.0 \AA$ resolution and had unit-cell parameters $a=b=82.49, c=62.47 \AA$, $\alpha=\beta=90$, $\gamma=120^{\circ}$. With one fusion-protein molecule in the asymmetric unit, the Matthews coefficient and solvent content were calculated to be $2.55 \AA^{3} \mathrm{Da}^{-1}$ and $51.84 \%$, respectively.

\section{Introduction}

Protein modification by covalent attachment of ubiquitin and ubiquitin-like molecules (UBLs) plays an essential role in the function and regulation of many molecular processes in all eukaryotic organisms (Goldberg, 2007; Schulman \& Harper, 2009). Three kinds of enzymes are involved in this activation cascade, including UBL activating enzyme (E1), UBL conjugation enzyme (E2) and UBL ligase (E3). Different UBLs have distinct E1s. Based on domain architecture, these E1s are divided into canonical and noncanonical E1s (Schulman \& Harper, 2009). Canonical E1s contain an inactive adenylation domain, an active adenylation domain, the first conserved catalytic cysteine domain $(\mathrm{FCCH})$, the second conserved catalytic cysteine domain ( $\mathrm{SCCH}$ ) and a C-terminal ubiquitin-fold domain. Noncanonical E1s comprise Uba4, Uba5 and Atg7, and they differ greatly from canonical E1s in molecular weight, domain architecture and the mechanisms underlying the activation of their corresponding UBLs.

Ubiquitin-fold modifier 1 (Ufm1) belongs to the UBL family with a dedicated E1 (Uba5), E2 (Ufc1) and E3 (Uf11) (Komatsu et al., 2004; Dou et al., 2005; Tatsumi et al., 2010). The Uba5-Ufm1 modification system is conserved in metazoans and plants, but not in yeasts, suggesting an important role in multicellular organisms (Komatsu et al., 2004; Gannavaram et al., 2011; Zhang et al., 2012; Hertel et al., 2013). Previous studies have shown that Uba5 is important for cell autonomous erythroid differentiation and is involved in the regulation of haematopoiesis (Tatsumi et al., 2011). Uba5 comprises only an adenylation domain (AD) followed by a C-terminal domain (CTD), with the canonical FCCH and SCCH domains being absent (Fig. 1a). The N-terminal residues (1-56) of Uba5 are not conserved among different species, and are unnecessary for Ufm1 activation (Zheng et al., 2008). The crystal structure of the Uba5 adenylation domain (residues 57-329; PDB entry 3h8v, Bacik et al., 2010) reveals that the adenylation domain is similar to that of its E1 homologues. Ufc1 is the downstream E2 for Uba5. Although Ufc1 shares low sequence 
identity with the canonical E2, their structures are topologically similar (Mizushima et al., 2007; Liu et al., 2009). Despite these advances, the mechanism underlying Ufc1 recognition by Uba5 and the subsequent formation of the Ufc1-Ufm1 complex remains ambiguous.

This study shows that the Uba5 C-terminal domain is necessary for the transthiolation of Ufm1 to Ufc1. The minimal region of Uba5 required for Ufc1 recognition was identified. The expression, purification, crystallization and preliminary X-ray crystallographic analysis of the fusion protein between Uba5 381-404 and Ufc1 are also reported. These results provide a basis for further understanding the molecular mechanisms of Uba5-mediated Ufc1 recognition and Ufm1 transthiolation.

\section{Materials and methods}

\subsection{Cloning}

Five constructs of Homo sapiens Uba5 (EC 6.3.2.19, NCBI accession No. NM_024818.3) were prepared in this study (Fig. 1a). Uba5 57-363, 57-404 and Ufc1 (EC 6.3.2.19, NCBI accession No. NP_057490.2) were amplified by PCR from cDNA plasmids, digested with the restriction endonucleases $N d e \mathrm{I}$ and $\mathrm{XhoI}$ (New England Biolabs) and ligated into the vector pETDuet-1 (Novagen). The GST- tagged Uba5 C-terminal fragments (330-404, 364-404 and 381-404) were amplified, digested with $N d e \mathrm{I}$ and $\mathrm{XhoI}$, and ligated into the vector pGEX-6P-1 (GE Healthcare). Empty pGEX-6P-1 vector was used for the expression of GST proteins. The Uba5 381-404 Ufc1 fusion protein was amplified using two-round PCR. In the first round, two primers (5'-CATGGCCAAAATGAAGAATATGGGCTCTGGCTCTGGCTCTGGCTCTATGGCGGATGAAGCCACGCGA-3' and 5'-TCGCGTGGCTTCATCCGCCATAGAGCCAGAGCCAGAGCCAGAGCCCATATTCTTCATTTTGGCCATG-3') were used to generate a fused Uba5-Ufc1 template linked by a four Gly-Ser repeat coding sequence. The fusion protein was then amplified by PCR using primers containing NdeI and XhoI sites. The second-round PCR products were digested and ligated into the vector pETDuet-1. The ligated products were then transformed into Escherichia coli $\mathrm{DH} 5 \alpha$ competent cells and the positive transformants were selected from agar plates containing $100 \mu \mathrm{g} \mathrm{ml}^{-1}$ ampicillin. The authenticity of the construct was confirmed by DNA sequencing.

\subsection{Expression and purification}

The recombinant plasmids for Uba5 57-363, Uba5 57-404, Ufc1, Uba5 381-404 Ufc1, Uba5 330-404, 364-404, 381-404 and empty

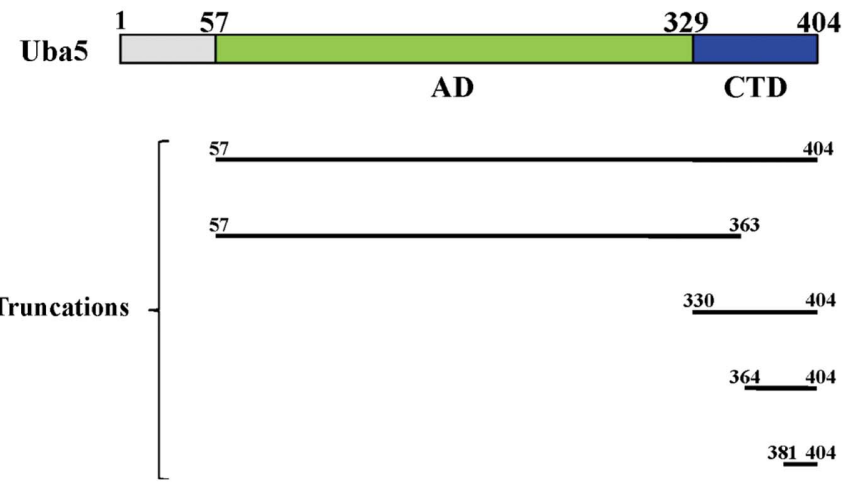

(a)

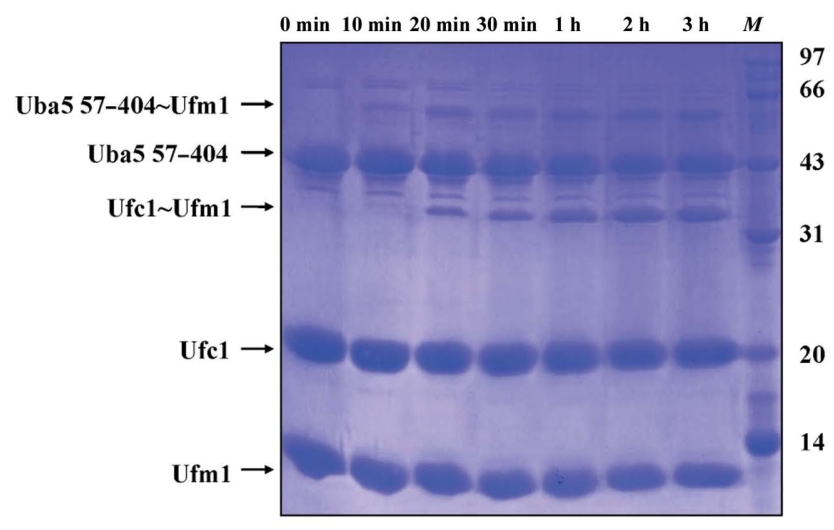

(c)

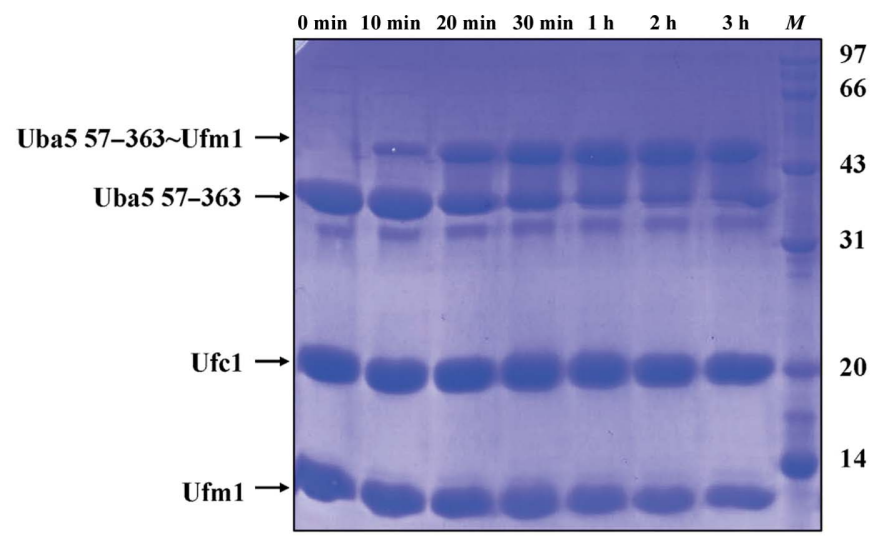

(b)

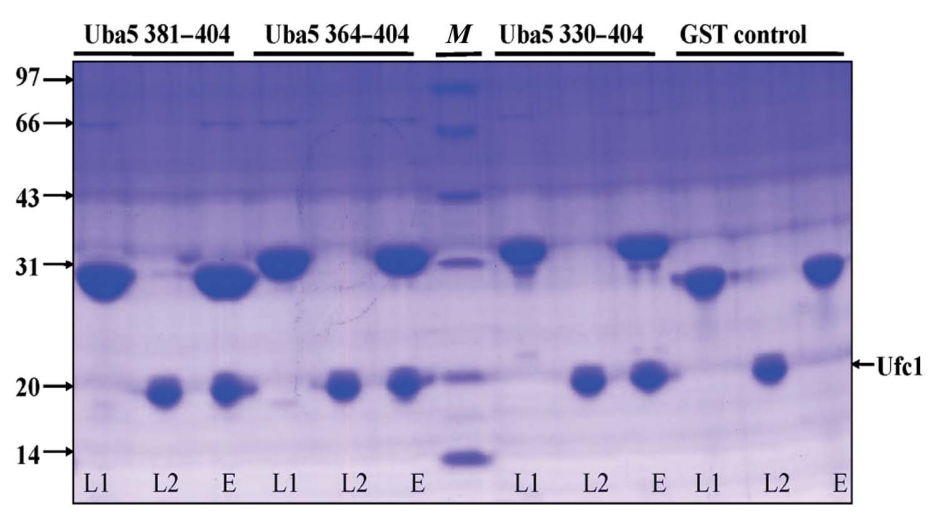

(d)

Figure 1

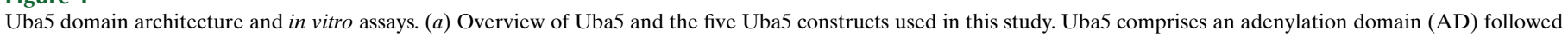

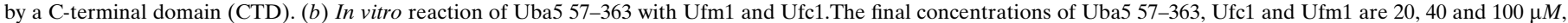

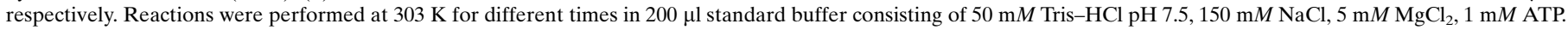

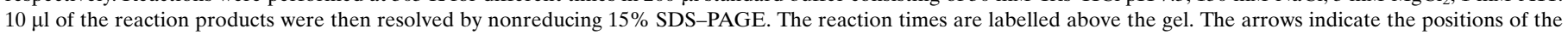

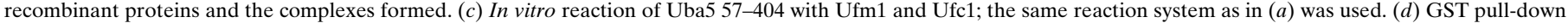

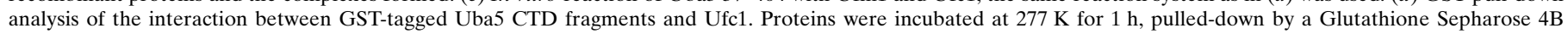

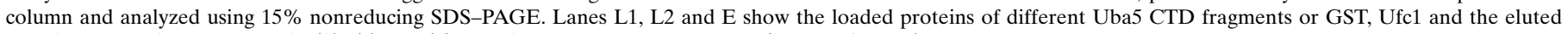
proteins, respectively. Lane $M$ in $(b),(c)$ and $(d)$ contains molecular-mass marker (labelled in $\mathrm{kDa}$ ). 
pGEX-6P-1 vector were transformed into E. coli BL21 (DE3) competent cells via a heat-shock procedure. The positive transformants were selected from agar plates containing $100 \mu \mathrm{g} \mathrm{ml}^{-1}$ ampicillin and were inoculated into $10 \mathrm{ml} \mathrm{LB}$ (Luria-Bertani) medium overnight at $310 \mathrm{~K}$ with agitation. The overnight cell culture was diluted into $11 \mathrm{LB}$ and grown under the same conditions to an $\mathrm{OD}_{600}$ of 0.6. Recombinant proteins were induced with $0.15 \mathrm{~m} M$ isopropyl $\beta$-D-1-thiogalactopyranoside (IPTG) at $291 \mathrm{~K}$ for $12 \mathrm{~h}$.

The bacterial cells were harvested by centrifugation and resuspended in lysis buffer $(50 \mathrm{~m} M$ Tris- $\mathrm{HCl} \mathrm{pH} 8.0,300 \mathrm{~m} M \mathrm{NaCl})$ supplemented with $10 \mathrm{~m} M \mathrm{MgCl}_{2}, 200 \mathrm{U} \mathrm{ml}^{-1}$ DNaseI and $1 \mathrm{~m} M$ phenylmethanesulfonyl fluoride (PMSF), and then disrupted by sonication. The cell lysate was centrifuged at $8000 \mathrm{~g}$ at $277 \mathrm{~K}$ for 45 min. For Uba5 57-363, Uba5 57-404, Ufc1 and Uba5 381404 Ufc1, the cell lysate was first loaded onto an Ni-NTA column (Qiagen) and then eluted with elution buffer $(50 \mathrm{~m} M$ Tris- $\mathrm{HCl} \mathrm{pH}$ 8.0, $250 \mathrm{~m} M$ imidazole). For Uba5 330-404, 364-404 and 381-404, the cell lysate was loaded onto a Glutathione Sepharose 4B column (GE Healthcare $)$ and then eluted with elution buffer $(50 \mathrm{~m} M$ Tris- $\mathrm{HCl} \mathrm{pH}$ 8.0, $10 \mathrm{~m} M \mathrm{GSH})$. The partially purified proteins were loaded onto a Source 15Q column (GE Healthcare) pre-equilibrated with $50 \mathrm{mM}$

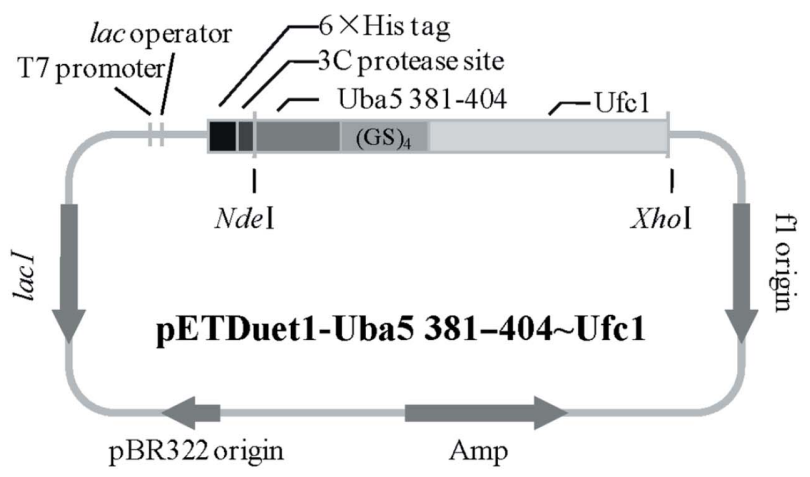

(a)

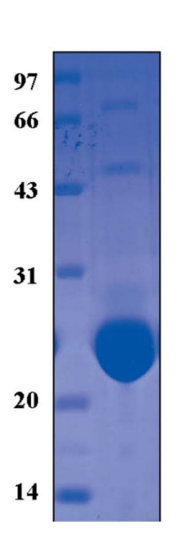

(b)

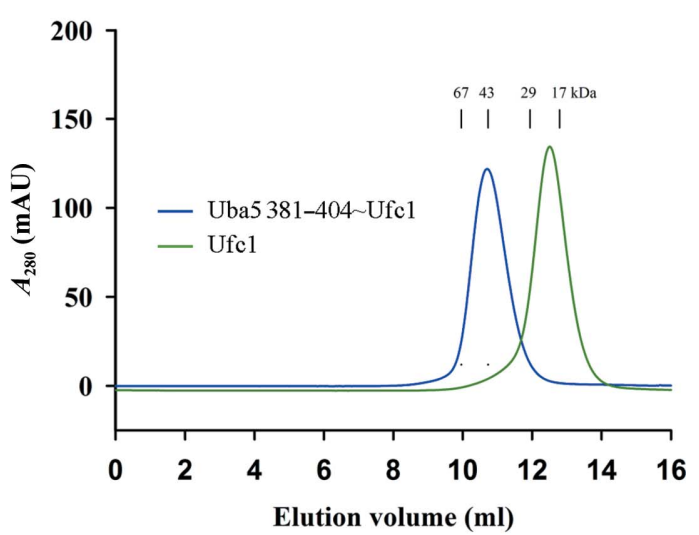

(c)
Figure 2

Cloning, expression and analysis of Uba5 381-404 Ufc1 fusion protein. (a) Expression vectors of the fusion protein. DNA fragments for the fusion protein were amplified and inserted into the pETDuet- 1 vector with an $\mathrm{N}$-terminal $6 \times \mathrm{His}$ tag for recombinant expression. (GS $)_{4}$ indicates the four Gly-Ser repeat linker region. (b) Purification of the recombinant protein. The purified proteins were resolved via 15\% SDS-PAGE, stained with Coomassie Brilliant Blue R-250 and then de-stained with an ethanol:acetic acid:water (2:1:7) mixture. The left lane contains the protein marker (labelled in $\mathrm{kDa}$ ) and the right lane contains $1.5 \mu \mathrm{l}$ of the purified recombinant fusion protein. (c) Oligomerization status of the fusion protein. The protein was analyzed using a Superdex 75 column. The standard markers indicated are 67, 43, 29 and $17 \mathrm{kDa}$.
Table 1

Data-collection and processing statistics for Uba5 381-404 Ufc1 fusion protein.

Values in parentheses are for the outer shell.

\begin{tabular}{ll}
\hline X-ray generator & Rigaku MicroMax-007 \\
Wavelength $(\AA)$ & 1.5419 \\
Temperature $(\mathrm{K})$ & 100 \\
Space group & $P 6_{2}$ or $P 6_{4}$ \\
$a, b, c(\AA)$ & $82.49,82.49,62.47$ \\
$\alpha, \beta, \gamma\left({ }^{\circ}\right)$ & $90.00,90.00,120.00$ \\
Mosaicity $\left({ }^{\circ}\right)$ & 0.33 \\
Resolution range $(\AA)$ & $19.81-3.00(3.21-3.00)$ \\
Total No. of reflections & 109990 \\
No. of unique reflections & 4926 \\
Completeness $(\%)$ & $99.7(100.0)$ \\
Multiplicity & $22.3(22.4)$ \\
$\langle I / \sigma(I)\rangle$ & $28.4(2.0)$ \\
CC $1 / 2$ & $1.000(0.865)$ \\
$R_{\text {r.i.m. }}$ & $0.106(1.879)$ \\
$R_{\text {p.i.m. }} R_{\text {merge }} \dagger(\%)$ & $0.022(0.397)$ \\
\hline
\end{tabular}

$\dagger R_{\text {merge }}=\sum_{h k l} \sum_{i}\left|I_{i}(h k l)-\langle I(h k l)\rangle\right| / \sum_{h k l} \sum_{i} I_{i}(h k l)$.

Tris- $\mathrm{HCl} \mathrm{pH} 8.0$ and then eluted using a linear gradient of $0-1 \mathrm{M}$ $\mathrm{NaCl}$. The fusion protein used for crystallization and Ufc1 used for the pull-down assay were treated with $3 \mathrm{C}$ protease (derived from rhinovirus) to remove the $6 \times$ His tag. Gly-Pro-His residues were left on the $\mathrm{N}$-terminus of the fusion protein from the vector cloning sites. GST proteins used as control in pull-down assays were expressed by empty pGEX-6P-1 vector and subsequently treated with $3 \mathrm{C}$ protease, with Leu-Glu-Val-Leu-Phe-Gln residues left on the C-terminus from the vector cloning sites. Fractions were then condensed and loaded onto a Superdex 75 (GE Healthcare) column equilibrated with $10 \mathrm{~m} M$ Tris- $\mathrm{HCl} \mathrm{pH}$ 8.0, $150 \mathrm{~m} M \mathrm{NaCl}$. Purified protein was analyzed by $12 \%$ SDS-PAGE and the protein concentration was determined by ultraviolet absorption at $280 \mathrm{~nm}$ (for recombinant Uba5 381404 Ufc1, $1 A_{280}=0.55 \mathrm{mg} \mathrm{ml}^{-1}$ ). The purified fusion proteins concentrated to $15 \mathrm{mg} \mathrm{ml}^{-1}$ were aliquoted, frozen in liquid nitrogen and stored at $193 \mathrm{~K}$ for further experiments and crystallization.

\subsection{In vitro reaction assay}

Activation and transfer of Ufm1 to Ufc1 by Uba5 were analyzed by incubating the indicated concentrations of Uba5, Ufc1 and Ufm 1 in a $200 \mu \mathrm{l}$ reaction system. The reaction was performed at $303 \mathrm{~K}$ for different durations, then stopped using SDS-PAGE sample buffer (80 m $M$ Tris- $\mathrm{HCl} \mathrm{pH} 6.8,2 \%$ SDS, $10 \%$ glycerol) and resolved by $15 \%$ nonreducing SDS-PAGE.

\subsection{GST pull-down assay}

Purified Ufc1 and GST-tagged Uba5 C-terminal fragments were mixed in a buffer consisting of $10 \mathrm{~m} M$ Tris- $\mathrm{HCl}$ pH 8.0, $150 \mathrm{~m} M \mathrm{NaCl}$ and incubated for $1 \mathrm{~h}$ at $277 \mathrm{~K}$. The mixtures were then loaded onto a Glutathione Sepharose 4B column and washed three times with incubating buffer. Subsequently, the bound proteins were eluted with elution buffer (50 m $M$ Tris- $\mathrm{HCl} \mathrm{pH}$ 8.0, $10 \mathrm{~m} M$ GSH) and analyzed by $15 \%$ nonreducing SDS-PAGE.

\subsection{Crystallization}

Crystallization of the Uba5 381-404 Ufc1 fusion protein was performed manually at $293 \mathrm{~K}$ via the hanging-drop method with different screening kits, including Crystal Screen, Index, PEG/Ion and SaltRx from Hampton Research and ProComplex Suite from Qiagen. 24-Well plates for crystallization (Beijing SeaskyBio Technology) were used with $1 \mathrm{ml}$ reservoir buffer. Small irregular crystals 


\section{crystallization communications}

appeared in drops comprising $1.5 \mu \mathrm{l}$ fusion-protein solution and $1.5 \mu \mathrm{l}$ reservoir solution consisting of $100 \mathrm{~m} M$ HEPES-NaOH pH 7.0, 12$15 \%(w / v)$ PEG 4000, 0.1-0.3 $\mathrm{M} \mathrm{KCl}$ after $2 \mathrm{~d}$ of growth. To improve $\mathrm{X}$-ray diffraction quality, the crystals were subsequently optimized by microseeding. Droplets consisting of fusion-protein and reservoir solutions mixed in a 1:1 ratio were pre-equilibrated for $30 \mathrm{~min}$ against $1 \mathrm{ml}$ reservoir solution. Several crystals that appeared in $100 \mathrm{mM}$ HEPES-NaOH pH 7.0, 13\%(w/v) PEG 4000, $0.16 M \mathrm{KCl}$ were scooped up using a $0.1 \mathrm{~mm}$ loop (Hampton Research) and then transferred into a tube containing $45 \mu \mathrm{l}$ seed-stock solution consisting of $100 \mathrm{~m} M$ HEPES-NaOH pH 7.0, 14\%( $w / v)$ PEG 4000, $0.2 M \mathrm{KCl}$. The crystals were broken up using a Seed Bead kit (Hampton Research) when vortex-mixed using $10 \times 5 \mathrm{~s}$ bursts. The seed stock was diluted with the seed-stock solution to 1:10, 1:100 and 1:1000 ratios. Subsequently, $0.2 \mu \mathrm{l}$ of seeds at the different dilutions were

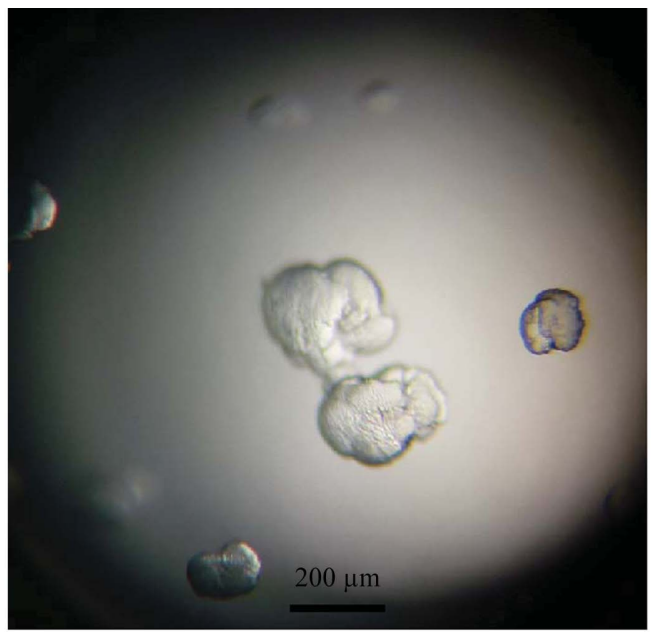

(a)

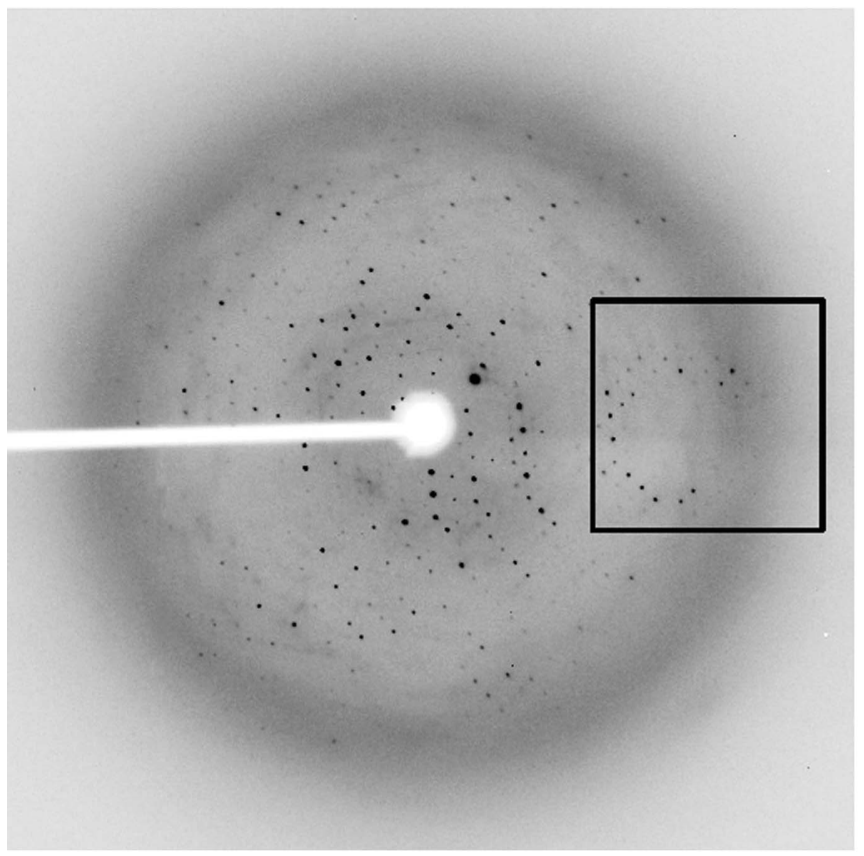

(c) added to the pre-equilibrated $3.0 \mu \mathrm{l}$ drops. The seed stocks were freshly prepared for each round of microseeding.

\subsection{Data collection and processing}

Fresh single crystals were harvested by transferring them with a cryoloop into cryoprotectant solution consisting of the reservoir solution supplemented with $25 \%(v / v)$ ethylene glycol and were then flash-cooled in liquid nitrogen. The diffraction data were collected inhouse using a Rigaku MicroMax-007 X-ray generator equipped with a Saturn 944 CCD detector. Data sets were collected at $100 \mathrm{~K}$ with an exposure time of $60 \mathrm{~s}$. The best crystal diffracted to $3.0 \AA$ resolution using a crystal-to-detector distance of $75 \mathrm{~mm}$ with $1^{\circ}$ oscillation per image. The complete data set was integrated using XDS (Kabsch, 2010) and scaled with AIMLESS (Evans, 2006).

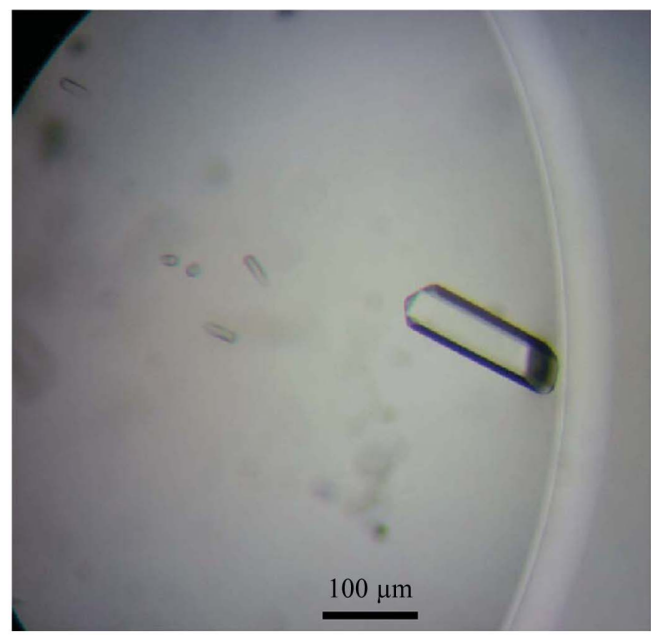

(b)

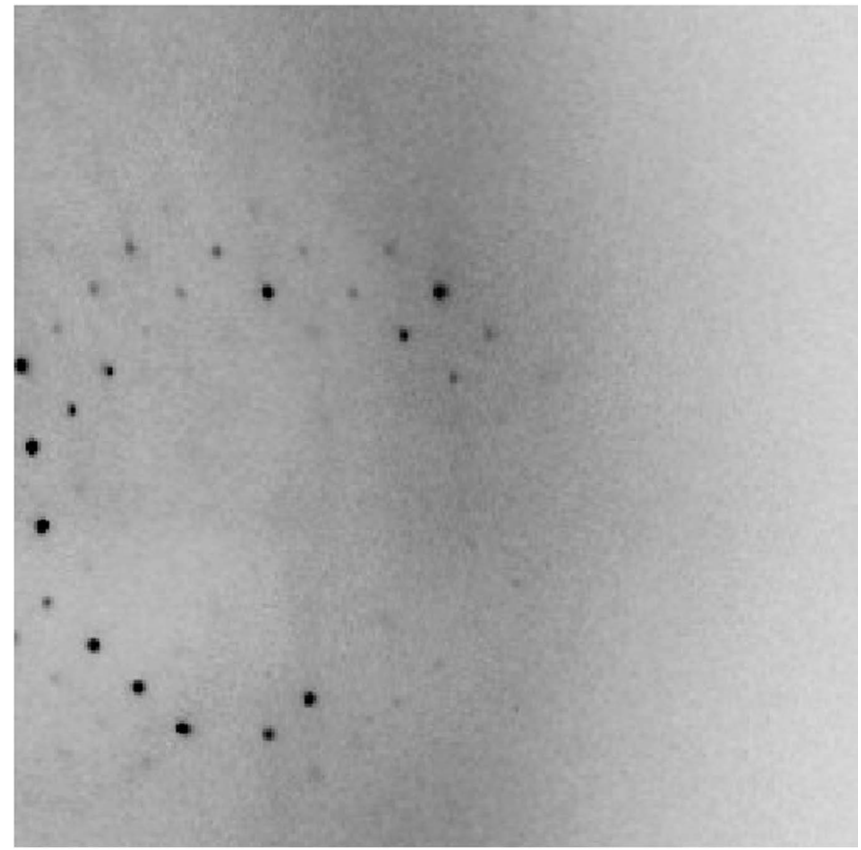

(d)

Figure 3

Preliminary X-ray crystallographic analysis of Uba5 381-404 Ufc1 fusion protein. (a) Crystals of the fusion protein. Crystals were obtained after $3 \mathrm{~d}$ at $293 \mathrm{~K}$ in crystallization buffer consisting of $100 \mathrm{~m} M$ HEPES-NaOH pH 7.0, $12-15 \%(w / v)$ PEG 4000, 0.1-0.3 $M$ KCl. (b) Optimized crystals by microseeding in $100 \mathrm{~m} M$ HEPES$\mathrm{NaOH} \mathrm{pH} \mathrm{7.0,12 \% (w/v)} \mathrm{PEG} \mathrm{4000,} 0.15 \mathrm{M} \mathrm{KCl}$, and $7 \mathrm{~d}$ of growth. $(c)$ Diffraction pattern of the crystal. $(d)$ Partial enlargement of the region in $(c)$ surrounded by the rectangle. 


\section{Results and discussion}

All canonical E1s possess a C-terminal ubiquitin-fold domain for recognition and recruitment of downstream E2s. In contrast, noncanonical E1s vary significantly in domain architecture, e.g. Atg7 recognizes Atg3 via an N-terminal domain consisting of a unique fold, which is located before the adenylation domain (Hong et al., 2011; Noda et al., 2011; Taherbhoy et al., 2011). Our previous studies showed that Uba5 57-363 is the minimal fragment necessary for highefficiency activation of Ufm1 (Xie, 2014). However, the molecular mechanism of Ufc1 recognition by Uba5 and the formation of the Ufc1-Ufm1 complex remain unknown.

To elucidate the functions of Uba5 364-404 in Ufm1 transthiolation, we conducted in vitro reactions using two truncations: Uba5 57363 and 57-404. The yields of Uba5 57-363, 57-404 and Ufc1 were about 60, 48 and $42 \mathrm{mg}$ per litre of culture, respectively. Uba5 57-363 failed to induce formation of the Ufc1-Ufm1 complex after $3 \mathrm{~h}$ of incubation in the system (Fig. 1b), while Uba5 57-404 leads to a comigrating band of the Ufc1-Ufm1 complex after 20 min of reaction (Fig. 1c). Both truncations retain the ability to bind efficiently to Ufm1 in the reaction system. These results suggest that Uba5 residues 364-404, which are at the C-terminus, have no influence on the activation of Ufm1. However, they are necessary for the transfer of the activated Ufm1 to Ufc1. Subsequently, GST pull-down assays were conducted with untagged Ufc1 and three different GST-tagged Uba5 C-terminal region fragments (Fig. 1d). The yield of GST-tagged Uba5 330-404, 364-404, 381-404 and GST was about 45, 39, 42 and $56 \mathrm{mg}$ per litre of culture, respectively. GST-tagged Uba5 330-404, 364-404 and 381-404 can strongly bind Ufc1, whereas GST alone cannot. This finding indicates that Uba5 381-404 is the minimal region for Ufc1 binding, which lies at the extreme C-terminal region of Uba5. Secondary-structural prediction reveals that this region probably forms an intact $\alpha$-helix (Xie, 2014). Sequence analysis shows that this fragment is enriched in hydrophobic residues, which implies that the Ufc1 binding may be facilitated by hydrophobic interactions.

To further uncover the molecular mechanism of Uba5-Ufc1 recognition, the Uba5 381-404 Ufc1 fusion protein was expressed and purified, with a yield of about $50 \mathrm{mg}$ per litre of culture, and its oligomerization status in solution was analyzed by gel-filtration chromatography (Fig. 2). The theoretical molecular mass of Uba5 381-404 was $2.66 \mathrm{kDa}$ and that of Ufc1 was $19.4 \mathrm{kDa}$. The fusion of Uba5 381-404 and Ufc1 with a four Gly-Ser repeat linker region was therefore $22.6 \mathrm{kDa}$ (Figs. $2 a$ and $2 b$ ). Ufc1 eluted as monomers, whereas the fusion protein eluted as a homodimer in the gel-filtration assay (Fig. 2c). Given that the Uba5 C-terminal domain can interact with Ufc1, the formation of the homodimer may result from the intermolecular interactions between two fusion-protein molecules. Crystallographic studies on the Uba5 381-404 Ufc1 fusion protein were then performed (Fig. 3). The initial crystals had an irregular shape and very weak diffraction (Fig. $3 a$ ). Through microseeding, single rod-like crystals were visible after $2 \mathrm{~d}$, and these crystals developed to their maximum dimensions, typically $100 \times 40 \times 40 \mu \mathrm{m}$, after $7 \mathrm{~d}$. High-quality crystals suitable for data collection were obtained after microseeding in $100 \mathrm{~m} M$ HEPES-NaOH $\mathrm{pH} 7.0$, $12 \%(w / v)$ PEG 4000, $0.15 \mathrm{M} \mathrm{KCl}$ for $7 \mathrm{~d}$ of growth (Fig. $3 b$ ).

$\mathrm{X}$-ray diffraction data were collected to $3.0 \AA$ resolution (Figs. $3 c$ and $3 d$ ). The space group was determined to be $P 6_{2}$ or $P 6_{4}$. The unit- cell parameters were $a=b=82.49, c=62.47 \AA, \alpha=\beta=90, \gamma=120^{\circ}$. With one fusion-protein molecule $(22.6 \mathrm{kDa})$ in the crystallographic asymmetric unit, the calculated Matthews coefficient ( $V_{\mathrm{M}}$; Matthews, $1968)$ is $2.55 \AA^{3} \mathrm{Da}^{-1}$ and the solvent content is $51.84 \%$. The data statistics are presented in Table 1. Phase determination was attempted by molecular replacement using Phaser (McCoy et al., 2007) with the structure of Ufc1 (PDB entry 2z6p; Mizushima et al., 2007) as the search model. However, it failed even after several attempts. This may be due to conformational changes of Ufc1 induced by the Uba5 C-terminal region. Additional attempts at phase determination are in progress using multi-wavelength anomalous diffraction (MAD) and heavy-atom soaking.

We thank Dr Masaaki Komatsu for generously providing the cDNA plasmid of human Uba5 and Ufc1. This work was supported by 31070643 and 31130062 (Natural Science Foundation of China), 2011CB910803 and 2013CB530603 (China National Key Basic Research Program).

\section{References}

Bacik, J. P., Walker, J. R., Ali, M., Schimmer, A. D. \& Dhe-Paganon, S. (2010). J. Biol. Chem. 285, 20273-20280.

Dou, T., Gu, S., Liu, J., Chen, F., Zeng, L., Guo, L., Xie, Y. \& Mao, Y. (2005). Mol. Biol. Rep. 32, 265-271.

Evans, P. (2006). Acta Cryst. D62, 72-82.

Gannavaram, S., Sharma, P., Duncan, R. C., Salotra, P. \& Nakhasi, H. L. (2011). PLoS One, 6, e16156.

Goldberg, A. L. (2007). Biochem. Soc. Trans. 35, 12-17.

Hertel, P., Daniel, J., Stegehake, D., Vaupel, H., Kailayangiri, S., Gruel, C., Woltersdorf, C. \& Liebau, E. (2013). J. Biol. Chem. 288, 10661-10671.

Hong, S. B., Kim, B.-W., Lee, K.-E., Kim, S. W., Jeon, H., Kim, J. \& Song, H. K. (2011). Nature Struct. Mol. Biol. 18, 1323-1330.

Kabsch, W. (2010). Acta Cryst. D66, 125-132.

Komatsu, M., Chiba, T., Tatsumi, K., Iemura, S., Tanida, I., Okazaki, N., Ueno, T., Kominami, E., Natsume, T. \& Tanaka, K. (2004). EMBO J. 23, $1977-$ 1986.

Liu, G., Forouhar, F., Eletsky, A., Atreya, H. S., Aramini, J. M., Xiao, R., Huang, Y. J., Abashidze, M., Seetharaman, J., Liu, J., Rost, B., Acton, T., Montelione, G. T., Hunt, J. F. \& Szyperski, T. (2009). J. Struct. Funct. Genomics, 10, 127-136.

Matthews, B. W. (1968). J. Mol. Biol. 33, 491-497.

McCoy, A. J., Grosse-Kunstleve, R. W., Adams, P. D., Winn, M. D., Storoni, L. C. \& Read, R. J. (2007). J. Appl. Cryst. 40, 658-674.

Mizushima, T., Tatsumi, K., Ozaki, Y., Kawakami, T., Suzuki, A., Ogasahara, K., Komatsu, M., Kominami, E., Tanaka, K. \& Yamane, T. (2007). Biochem. Biophys. Res. Commun. 362, 1079-1084.

Noda, N. N., Satoo, K., Fujioka, Y., Kumeta, H., Ogura, K., Nakatogawa, H., Ohsumi, Y. \& Inagaki, F. (2011). Mol. Cell, 44, 462-475.

Schulman, B. A. \& Harper, J. W. (2009). Nature Rev. Mol. Cell Biol. 10, 319 331.

Taherbhoy, A. M., Tait, S. W., Kaiser, S. E., Williams, A. H., Deng, A., Nourse, A., Hammel, M., Kurinov, I., Rock, C. O., Green, D. R. \& Schulman, B. A. (2011). Mol. Cell, 44, 451-461.

Tatsumi, K., Sou, Y.-S., Tada, N., Nakamura, E., Iemura, S., Natsume, T., Kang, S. H., Chung, C. H., Kasahara, M., Kominami, E., Yamamoto, M., Tanaka, K. \& Komatsu, M. (2010). J. Biol. Chem. 285, 5417-5427.

Tatsumi, K., Yamamoto-Mukai, H., Shimizu, R., Waguri, S., Sou, Y.-S., Sakamoto, A., Taya, C., Shitara, H., Hara, T., Chung, C. H., Tanaka, K., Yamamoto, M. \& Komatsu, M. (2011). Nature Commun. 2, 181.

Xie, S. (2014). Acta Cryst. F70, 765-768.

Zhang, Y., Zhang, M., Wu, J., Lei, G. \& Li, H. (2012). PLoS One, 7, e48587.

Zheng, M., Gu, X., Zheng, D., Yang, Z., Li, F., Zhao, J., Xie, Y., Ji, C. \& Mao, Y. (2008). J. Cell. Biochem. 104, 2324-2334. 